hep-th/0102138

$\mathrm{IP} / \mathrm{BBSR} / 2001-03$

\title{
Holography and Stiff-matter on the Brane
}

\author{
Anindya K. Biswas[ and Sudipta Mukherjif] \\ Institute of Physics, \\ Bhubaneswar 751 005, INDIA
}

\begin{abstract}
Recently, Verlinde noted a surprising similarity between Friedmann equation governing radiation dominated universe and Cardy's entropy formula in conformal field theory. In this note, we study a brane-universe filled with radiation and stiff-matter. We analyze Friedmann equation in this context and compare our results with Cardy's entropy formula.
\end{abstract}

February 2001

\footnotetext{
${ }^{1}$ e-mail: anindya@iopb.res.in

${ }^{2}$ e-mail: mukherji@iopb.res.in
} 
Since 't Hooft [1] and Susskind [2] proposed the idea of holography, there have been several works in the past trying to test this proposal in various gravity systems. According to the holographic principle, dynamics of $D$ space-time dimensions are encoded in terms of the degrees of freedom on the $D-2$ dimensional boundary. The ADS/CFT correspondence does indeed realize such a principle. In systems where gravity is dynamical, testing holographic principle, however, turned out to be somewhat subtle. Fischler and Susskind [3], in particular, addressed the issue of holographic entropy bound in the cosmological setting. They proposed that the area of particle horizon should bound the entropy on the backward looking light-cone. However, violation of such bound was found in case of closed FRW universe. Subsequently, various modifications [四] were proposed to circumvent such a problem.

In a more recent paper [7], Verlinde analyzed entropy bound in a radiation dominated closed FRW universe using the holographic principle. Representing radiation as conformal field theory (CFT) in the universe, he found surprising similarity between Cardy's entropy formula for $1+1$ dimensional CFT and the Friedmann equation governing the evolution of the universe. In fact, it turned out after suitable identifications, that Cardy's formula maps to Friedmann equation. Furthermore, in [7], a cosmological entropy bound is proposed which unites Beckenstein bound [5] for limited self-gravity system and Hubble bound [6] for strong self-gravity system in an elegant way. 5 Though, Verlinde's analysis was for radiation dominated universe (which can be modeled perhaps by CFT), the arguments of the paper, leading to entropy bound, depend on the equation of state of matter in a very minimal way. This might lead one to suspect that a version of Verlinde's bound might hold even when the universe is described by a different equation of state. The purpose of this note is to test Verlinde's proposal for an universe filled with radiation and stiff matter. Following closely [12], we consider a brane-world in a charged black hole background [13]. The time development of the metric on the brane is described by Friedmann equation [9, 12]. The mass of the black hole background induces radiation matter on the brane [12]. On the other hand, the charge of the black hole, however, acts as a source for stiff matter on the brane ( whose equation of state is simply given by the pressure being equal to the energy density). We show that when the brane crosses the horizon of the charged black hole, entropy and temperature can be expressed in terms of the Hubble parameter and its derivative. In particular, none of these thermodynamical quantities depend explicitly on mass and charge of the background. This is similar to what was observed in [12], when the target space was uncharged and hence the brane is described by radiation dominated matter only. We also, some what surprisingly, find that the formula for entropy given in [12], from the CFT consideration, coincides with the FRW equation when the brane crosses the horizon of the charged black hole.

We start by considering an $(n+1)$ dimensional brane in a space-time described by an $(n+2)$ dimensional charged AdS black hole. The background metric is thus given

\footnotetext{
${ }^{3}$ See also [8] for further work on analyzing Verlinde's proposal in different circumstances.
} 
by 13

$$
d s_{(n+2)}^{2}=-h(a) d t^{2}+\frac{d a^{2}}{h(a)}+a^{2} d \Omega_{n}^{2}
$$

where

$$
h(a)=1-\frac{\omega_{n+1} M}{a^{n-1}}+\frac{n \omega_{n+1}^{2} Q^{2}}{8(n-1) a^{2 n-2}}+\frac{a^{2}}{L^{2}} .
$$

Here, $M$ and $Q$ are respectively ADM mass and charge, $L$ is the curvature radius of space and $\omega_{n+1}=16 \pi \mathbf{G}_{N} /\left(n \operatorname{Vol}\left(S^{n}\right)\right)$. $\mathbf{G}_{\mathbf{N}}$ here is the Newton's constant. If $a_{H}$ is the largest real positive root of $h(a)$, then in order for (1) to describe a charged black hole with non singular horizon at $a=a_{H}$, the later should satisfy

$$
\left(\frac{n}{n-1}\right) a_{H}^{2 n}+L^{2} a_{H}^{2 n-2} \geq \frac{n L^{2} \omega_{n+1}^{2} Q^{2}}{8(n-1)} .
$$

The electrostatic potential difference between the horizon and infinity is given by the quantity $\Phi$ and following [13] we choose it to be

$$
\Phi=\left(\frac{n}{4 n-4}\right) \frac{\omega_{n+1} Q}{a^{n-1}} .
$$

This space-time was analyzed with a focus on AdS/CFT correspondence in [13]. 目

Let us now consider a $(n+1)$-dimensional brane with a constant tension in the background of (1). As discussed in [12], if we regard the brane as a boundary of background AdS geometry, location and the metric on the boundary becomes time dependent. The induced equation of motion of the metric on the brane satisfy Friedmann equation.

Tuning the brane cosmological constant to zero, we get the following induced equation on the brane:

$$
H^{2}=-\frac{1}{a^{2}}+\frac{\omega_{n+1} M}{a^{n+1}}-\frac{n \omega_{n+1}^{2} Q^{2}}{8(n-1) a^{2 n}}
$$

where $H=\frac{\dot{a}}{a}$ being the Hubble parameter and dot denotes derivation with respect to the brane time $\tau$. Someone who is living on the brane will thus observe a closed FRW metric given by

$$
d s_{n+1}^{2}=-d \tau^{2}+a(\tau)^{2} d \Omega_{n}^{2}
$$

with $a(\tau)$ satisfying (5). Now from different power law behaviour of the quantities in the right hand side of (5) with respect to $a$, we conclude that while $M$ behaves as

\footnotetext{
${ }^{4}$ Interestingly enough, it was found that in the high temperature limit the charge essentially plays no role (see the discussion in the last section of [13]). So at least in this limit the CFT that lives in the boundary is expected to behave in the same manner as was discussed by Witten [14.

5 The way to arrive at such equation has been discussed in [9] 12], we will skip the detail here.
} 
a source of radiation matter as in [12], $Q$ behaves as a source of stiff matter on the brane. However, we should notice that the $Q^{2}$ dependent term in (5) has appeared with a wrong sign in front. Differentiating (5) with respect to $\tau$ we get

$$
\dot{H}=\frac{1}{a^{2}}-\frac{(n+1) \omega_{n+1} M}{2 a^{n+1}}+\frac{n^{2} \omega_{n+1}^{2} Q^{2}}{8(n-1) a^{2 n}} .
$$

At this point, we would like to comment on the cosmological behaviour of the universe on the brane. The time development of the brane-universe is completely determined by (5). Like closed model for radiation dominated universe, brane-universe reaches a maximum value $a_{\max }$ which is given by the largest real root of the equation,

$$
\frac{1}{a^{2}}+\frac{\omega_{n+1} M}{a^{n+1}}-\frac{n \omega_{n+1}^{2} Q^{2}}{8(n-1) a^{2 n}}=0
$$

Furthermore, it is easy to check that there is a non-zero minimum value for the scale factor $a_{\min }$. So the brane-universe oscillates between $a_{\min }$ and $a_{\max }$. It can also easily be seen that $a_{\min }<a_{H}<a_{\max }$, where $a_{H}$ is the location of the horizon of the black hole background given in (11). Now, if we compare (8) with (5), we see that the Hubble parameter satisfy $H^{2}=\frac{1}{L^{2}}$. This is similar to what was found in [12]. We also note that $H$ is independent of black hole parameters $M$ and $Q$ at the horizon.

If we take the brane as the boundary of the AdS space, following [14], one would expect that the entropy of the CFT is given by the Bekenstein-Hawking entropy of the charged AdS black hole

$$
S=\frac{a_{H}^{n} \operatorname{Vol}\left(S^{n}\right)}{4 \mathbf{G}_{N}}
$$

From above, it follows that the entropy density on the brane is

$$
s=(n-1) \frac{a_{H}^{n}}{4 G_{N} L a^{n}}, \text { and at } a=a_{H}, s=\frac{(n-1) H}{4 G_{N}},
$$

where $G_{N}$ is the Newton's constant on the brane. It is related to $\mathbf{G}_{N}$ via $G_{N} L=$ $(n-1) \mathbf{G}_{N}$. These expressions are the same as that obtained in [12] where, the bulk space-time was an uncharged black hole. These expression are some what universal as they do not explicitly depend upon black hole parameters like mass and charge etc. Similarly, from the expression of the Hawking temperature for the metric in (11), one can obtain the induced temperature on the brane, which again does not depend explicitly on black hole parameters at $a=a_{H}$ and can be expressed as:

$$
T=-\left.\frac{\dot{H}}{2 \pi H}\right|_{a=a_{H}}\left(\frac{L}{a}\right) .
$$

When the brane crosses the horizon, we notice that all the thermodynamical quantities on the brane can be written down solely in terms of $H$ and $\dot{H}$. 
The first law of thermodynamics, written in terms of densities, takes the following form on the brane:

$$
T d s=d \rho-\Phi d \tilde{\rho}+n(\rho+p-\Phi \tilde{\rho}-T s) \frac{d a}{a} .
$$

Here, $\rho$ is the energy density, $p$ is the pressure, and $\tilde{\rho}$ is the charge density. The quantity $(\rho+p-\Phi \tilde{\rho}-T s)$ is a measure of non-extensiveness of thermodynamical quantities. Now using the identification,

$$
\begin{aligned}
\rho & =\frac{M L}{a^{n+1} \operatorname{Vol}\left(S^{n}\right)}, \quad p=\frac{M L}{n a^{n+1} \operatorname{Vol}\left(S^{n}\right)}, \\
\Phi & =\left(\frac{n}{4 n-4}\right) \frac{\omega_{n+1} Q L}{a^{n}}, \quad \tilde{\rho}=\frac{Q}{a^{n} \operatorname{Vol}\left(S^{n}\right)},
\end{aligned}
$$

we get

$$
\frac{n}{2}(\rho+p-\Phi \tilde{\rho}-T s)=\frac{\gamma}{a^{2}}
$$

where

$$
\gamma=\frac{n(n-1)}{16 \pi G_{N}} \frac{a_{H}^{n-1}}{a^{n-1}} .
$$

Following the same line arguments as [12], we can express the entropy density in the form of Cardy's formula

$$
s=\left(\frac{4 \pi}{n}\right) \sqrt{\gamma\left(\rho-\frac{\Phi \tilde{\rho}}{2}-\frac{\gamma}{a^{2}}\right)} .
$$

It is easy now to check that the equations (17) and (15) are the same as Friedmann equations (5) and (7) at $a=a_{H}$. When expressed in terms of total entropy, (17) reduces to the one discussed in [7].

Appearance of the quantity $\Phi \tilde{\rho}$ in (12), (15) and (17) is interesting. From the point of view of background black hole geometry, it measures the energy density due to charge. On the other hand, we expect that from the brane perspective, it should be the contribution of the energy density and pressure (which are equal in magnitude however) of stiff matter. It is satisfying to observe that

$$
\Phi \tilde{\rho}=\rho_{\mathrm{sm}}+p_{\mathrm{sm}} \text { at } a=a_{H},
$$

where subscripts on $\rho$ and $p$ indicate that they are stiff-matter contributions. We would like to emphasise that it is at $a=a_{H}$, where the entropy formula (17) and (15) coincides with Friedmann equation (5) and (7) respectively.

\section{Acknowledgements}

We would like to thank A. Kumar, B. Rai and A. Srivastava for general discussions on the holographic principle. SM would like to thank P. Parashar for her support during the course of this work. 


\section{References}

[1] G. 't Hooft, Abdus Salam Festschrift: a collection of talks, A. Ali, J. Ellis and S. Randjbar-Daemi (World Scientific, Singapore, 1993), gr-qc/9310026.

[2] L. Susskind, J. Math. Phys. 36 (1995) 6377, hep-th/9405069

[3] W. Fischler and L. Susskind, Holography and Cosmology, hep-th/9806039.

[4] R. Bousso, JHEP 07 (1999) 004, hep-th/9911002; G. Veneziano, Phys. Lett. B454 (1999) 22, hep-th/9907012; R. Easther and D.A. Lowe, Phys. Rev. Lett. 82 (1999) 4967, hep/th 9902088; D. Bak and S.J. Rey, Class. Quant. Grav. 17 (2000) L83, hep-th/9902173; S.K. Rama, Phys. Lett. B457 (1999) 268, hep-th/9904110; N. Kaloper and A. Linde, Phys. Rev. D60 (1999) 103509, hep-th/9904120.

[5] J.D. Bekenstein, Phys. Rev. D23 (1981) 287.

[6] G. Veneziano, Phys. Lett. B454 (1999) 22, R. Brustein and G. Veneziano, Phys. Rev. Lett. 84 (2000) 5695.

[7] E. Verlinde, On the holographic principle in a radiation dominated universe, hepth/0008140.

[8] D. Kutasov and F. Larsen, JHEP 01 (2001) 001, hep-th/0009244; F.-L. Lin, hep-th/0010127; S. Nojiri and S. Odintsov, hep-th/0011115; B. Wang, E. Abdalla and R.-K. Su, hep-th/0101073; D. Klemm, A. Petkou and G. Siopsis, hepth/0101076; R. Brustein, S. Foffa and G. Veneziano, hep-th/0101083; R.-G Cai, hep-th/0102113.

[9] S. Gubser, AdS/CFT and gravity, hep-th/9912001.

[10] C. Barcelo amd M. Visser, Phys. Lett. B482 (2000) 183, hep-th/0004056.

[11] C. Csaki, J. Erlich and C. Grojean, hep-th/0012143.

[12] I. Savonije and E. Verlinde, CFT and entropy on the brane, hep-th/0102042.

[13] A. Chamblin, R. Emparan, C. Johnson and R. Myers, Phys. Rev. D60 (1999) 064018, hep-th/9902170; R.-G. Cai and K.-S.Soh, Phys. Rev. D59 (1999) 044013, gr-qc/9808067.

[14] E. Witten, Adv. Theor. Math. Phys. 2 (1998) 253, hep-th/9802150. 